\title{
Structural Variant in Mitochondrial-Associated Gene (MRPL3) Induces Adult-Onset Neurodegeneration with Memory Impairment in the Mouse
}

\author{
Lindsay S. Cahill, ${ }^{1}$ Jessie M. Cameron, ${ }^{2}$ Julie Winterburn, ${ }^{1}$ Patrick Macos, ${ }^{1}$ Johnathan Hoggarth, ${ }^{1}$ \\ Misko Dzamba, ${ }^{3}$ Michael Brudno, ${ }^{3,4}$ Lauryl M. J. Nutter, ${ }^{5,6}$ Thomas J. Sproule, ${ }^{7}$ Robert W. Burgess, ${ }^{7}$ \\ ${ }^{\circledR}$ R. Mark Henkelman, ${ }^{1,8,9}$ and ${ }^{\circledR}$ John G. Sled ${ }^{1,8,9}$ \\ ${ }^{1}$ Mouse Imaging Centre, Hospital for Sick Children, Toronto, Ontario M5T 3H7, Canada, ${ }^{2}$ Metabolism Research Programme, Hospital for Sick \\ Children, Toronto, Ontario M5G 1X8, Canada, ${ }^{3}$ Department of Computer Science, University of Toronto, Toronto, Ontario M5T 3A1, Canada, \\ ${ }^{4}$ Centre for Computational Medicine, Hospital for Sick Children, Toronto, Ontario M5G 1X8, Canada, ${ }^{5}$ The Centre for Phenogenomics, Toronto, \\ Ontario M5T 3H7, Canada, ${ }^{6}$ Hospital for Sick Children, Toronto, Ontario M5G 1X8, Canada, ${ }^{7}$ The Jackson Laboratory, Bar Harbor, Maine 04609, \\ ${ }^{8}$ Translational Medicine, Hospital for Sick Children, Toronto, Ontario M5G 1X8, Canada, and ${ }^{9}$ Department of Medical Biophysics, University of \\ Toronto, Toronto, Ontario M5G 1L7, Canada
}

An impediment to the development of effective therapies for neurodegenerative disease is that available animal models do not reproduce important clinical features such as adult-onset and stereotypical patterns of progression. Using in vivo magnetic resonance imaging and behavioral testing to study male and female decrepit mice, we found a stereotypical neuroanatomical pattern of progression of the lesion along the limbic system network and an associated memory impairment. Using structural variant analysis, we identified an intronic mutation in a mitochondrial-associated gene $(\mathrm{Mrpl3})$ that is responsible for the decrepit phenotype. While the function of this gene is unknown, embryonic lethality in Mrpl3 knock-out mice suggests it is critical for early development. The observation that a mutation linked to energy metabolism precipitates a pattern of neurodegeneration via cell death across disparate but linked brain regions may explain how stereotyped patterns of neurodegeneration arise in humans or define a not yet identified human disease.

Key words: magnetic resonance imaging; mouse model; MRPL3; neurodegenerative disease

Significance Statement

The development of novel therapies for adult-onset neurodegenerative disease has been impeded by the limitations of available animal models in reproducing many of the clinical features. Here, we present a novel spontaneous mutation in a mitochondrial-associated gene in a mouse (termed decrepit) that results in adult-onset neurodegeneration with a stereotypical neuroanatomical pattern of progression and an associated memory impairment. The decrepit mouse model may represent a heretofore undiagnosed human disease and could serve as a new animal model to study neurodegenerative disease.

Received Jan. 3, 2020; revised Apr. 14, 2020; accepted Apr. 16, 2020.

Author contributions: L.S.C., M.B., L.M.J.N., T.J.S., R.W.B., R.M.H., and J.G.S. designed research; L.S.C., J.M.C., J.W., P.M., J.H., M.D., L.M.J.N., and T.J.S. performed research; L.S.C., M.D., and T.J.S. analyzed data; L.S.C. wrote the paper.

The authors declare no competing financial interests.

This work was supported by Canadian Institutes of Health Research Grant FDN-152961. We thank the Model Production and Clinical Phenotyping Cores at The Center for Phenogenomics (headed by Marina Gertsenstein and Ann Flenniken, respectively) for ES cell targeting, chimera generation, embryo dissections, and early adult phenotyping; and the Genome Technologies Service at The Jackson Laboratory for DNA capture and next-generation sequencing.

Correspondence should be addressed to Lindsay S. Cahill at Icahill@mun.ca.

https://doi.org/10.1523/JNEUROSCI.0013-20.2020

Copyright $\odot 2020$ the authors

\section{Introduction}

The identification of rare highly penetrant genetic mutations that cause familial forms of neurodegenerative disease has advanced the study of conditions such as Alzheimer's disease (Tanzi, 2012), Parkinson's disease (Klein and Westenberger, 2012), and amyotrophic lateral sclerosis (Ghasemi and Brown, 2018) by enabling the creation of genetically engineered mouse models. However, it has been difficult to create mouse models that accurately reproduce the adult-onset and progression that is observed in sporadically occurring human neurodegenerative disease. Moreover, as it remains unknown why these diseases exhibit stereotyped neuroanatomical patterns of progression, this aspect of these diseases has been difficult to study or model. 
Previously, a mouse model, termed "decrepit" $(d c r)$, arose from a spontaneous mutation in an inbred mouse colony and had been reported to have an adult-onset neurodegenerative disease (Sproule et al., 2010); however, the causal mutation and the mechanism of brain injury remained elusive. The $d c r$ phenotype becomes apparent at $\sim 10$ weeks of age (equivalent to adulthood in humans (Dutta and Sengupta, 2016)), when the mice develop an unkempt appearance and exhibit an abnormally hunched posture. The mice cease to gain weight and have a shortened life span compared with controls (18-22 weeks compare with $>52$ weeks). Histologic evaluation of the brain of $d c r$ mice revealed bilaterally symmetric loss of neurons and complete vacuolization of tissue in the cortex and hippocampus (Sproule et al., 2010). Dilated blood vessels and signs of stress reactions (GFAP-positive reactive astrocytes) were also observed; however, there was no evidence of hemorrhage or blood clots. The physiological basis for the cerebral pathology could not be determined.

Using advanced in vivo magnetic resonance imaging (MRI) in combination with deformation-based morphometry, we studied the progression of neurodegeneration and performed a comprehensive assessment of the changes in neuroanatomy. Compared with the histologic techniques used previously (Sproule et al., 2010), 3D MRI provides the advantage of unbiased, whole-brain analysis without tissue-processing artifacts. The $d r r$ phenotype was further investigated using the Barnes maze as a test of learning and memory, and using $\mathrm{x}$-ray micro-computed tomography (micro-CT) imaging to study cross-sectionally the alterations in cerebrovascular architecture. We identified a stereotypical neuroanatomical pattern of disease progression and associated memory impairment in decrepit mice. Using variant analysis of next-generation genome sequencing data, we were able to identify an intronic mutation in a largely unstudied gene responsible for the decrepit phenotype.

\section{Materials and Methods}

Animals. Mice carrying the $d c r$ mutation (C57BL/6J background; Sproule et al., 2010) were acquired from The Jackson Laboratory and bred at The Center for Phenogenomics (TCP). The colony was maintained by breeding mice that are heterozygous for the $d c r$ mutation, and the wild-type mice (without the $d c r$ mutation) were used as controls. The genotyping was completed using standard PCR and electrophoresis at The Center for Applied Genomics. The wild-type primers are forward primer (TCTCGAGAGTCAGCTCATAGAGACA) and reverse primer (CCCCGCAGAGACTCATTACCT), product size $451 \mathrm{bp}$; and the $d c r$ primer is forward primer (CAGGAACACCTCGATGCTC), produce size 214 bp. Mrpl3 (mitochondrial ribosomal protein L3) knock-out mice (C57BL/6N-Mrpl3 $\left.3^{\text {tm } 4 b(K O M P) T c p} / 2 \mathrm{Tcp}\right)$ were made as part of the North American Conditional Mouse Mutagenesis 2 (NorCOMM2) project and were generated using the Knockout Mouse Project (KOMP) targeting vector (PRPGS00036_D_A05) obtained from the KOMP Repository. The vector was used for targeting in C57BL/6NTac-C2 embryonic stem (ES) cell lines using standard protocols (Behringer et al., 2014), expanded and aggregated for chimera generation (Gertsenstein et al., 2010). Male chimeras with $>50 \%$ ES cell contribution to the coat color were bred with albino outbred CD-1 females. ES cell germline transmission was detected by black eyes and the coat color of the pups, then the chimera-transmitter was mated to $\mathrm{C} 57 \mathrm{BL} / 6 \mathrm{NCrl}$ mice and their progeny were screened. The ES cells and derived mouse line were subjected to quality control including long-range PCR across the 5' homology arm and quantitative real-time PCR (qRT-PCR) loss-of-allele assay for the wild-type allele [copy number $(\mathrm{CN})=1$ in correctly targeted mutations and $\mathrm{CN}=2$ in wild-type] to confirm targeting. Gene-specific short-range PCR to confirm the presence of the lacZ cassette and distal loxP sites and qRT-PCR to assess vector copy number $(\mathrm{CN}=1)$ were also done (data not shown). Cre excision was achieved by breeding a heterozygous C57BL/6N-Mrpl3 ${ }^{\operatorname{tm} 4 a(K O M P) T c p} / 2 \mathrm{Tcp}$ male with heterozygous C57BL/
Table 1. Number of mice included in the analysis for each experimental assay

\begin{tabular}{|c|c|c|c|c|c|c|}
\hline \multirow{2}{*}{$\begin{array}{l}\text { Experimental } \\
\text { Assay }\end{array}$} & \multicolumn{2}{|c|}{$\begin{array}{l}\text { Control } \\
\text { mice }\end{array}$} & \multicolumn{2}{|c|}{$\begin{array}{l}d c r \\
\text { mice }\end{array}$} & \multicolumn{2}{|c|}{$\begin{array}{c}{\mathrm{Mrp} / 3^{+1-}}^{\text {mice }} \\
\text { mich }\end{array}$} \\
\hline & M & $\mathrm{F}$ & M & $\mathrm{F}$ & M & $\mathrm{F}$ \\
\hline In vivo $M R$ imaging & 17 & 20 & 23 & 14 & & \\
\hline Barnes maze testing & 5 & 4 & 5 & 4 & & \\
\hline Ex vivo micro-CT imaging & 5 & 8 & 6 & 11 & & \\
\hline Mitochondrial enzyme activity & 9 & 7 & 10 & 5 & & \\
\hline IMPReSS phenotyping & 10 & 9 & & & 10 & 9 \\
\hline Ex vivo $M R$ imaging & 10 & 9 & & & 12 & 12 \\
\hline
\end{tabular}

F, Female; M, male.

$6 \mathrm{~N}-G t(R O S A) 26$ Sor $^{t m 1(A C T B-C r e,-E G F P) I c s} / \mathrm{Tcp}$ (Cre-EGFP) females to produce $\mathrm{C} 57 \mathrm{BL} / 6 \mathrm{~N}-\mathrm{Mrpl3} 3^{\operatorname{tm} 4 b(K O M P) T c p} / 2 \mathrm{Tcp}$ mice. The mouse line was established by backcrossing $M r p l 3^{t m 4 b+l-}$;Cre-EGFP' mice to C57BL/ 6NCrl (Charles River Laboratories). Heterozygotes were intercrossed and sampled for genotyping at postnatal day 14 (P14) to P17. Lethality was defined as the absence of homozygous mice after screening at least 28 pups (Dickinson et al., 2016; 99 pups genotyped during adult phenotyping cohort production for early adult phenotyping (see below); 27 wild-type; 72 heterozygotes; 0 homozygotes). All animal experiments were approved by the Animal Care Committee at The Center for Phenogenomics and performed in accordance with guidelines established by the Canadian Council on Animal Care.

Experimental design and statistical analyses. All statistical tests were performed using RMINC (https://github.com/Mouse-Imaging-Centre/ RMINC) and R statistical software (www.r-project.org). Table 1 provides a detailed description of animal allocation within the study. Each assay was conducted on a separate cohort of mice. To assess lesion progression and to detect changes in neuroanatomy associated with the $d c r$ mutation, $37 \mathrm{dcr}$ mice (23 males and 14 females) and 37 control mice (17 males and 20 females) were imaged cross-sectionally using in vivo MRI from 50 to $150 \mathrm{~d}$ of age. The recovery rate of the $\mathrm{dcr}$ mice after prolonged isoflurane exposure $(>2.5 \mathrm{~h})$ was low, and therefore the mice could only be imaged at one time point. To assess whether there were differences in the trajectory of brain growth between $\mathrm{dcr}$ and control mice, we used a best fit linear or quadratic model (a quadratic model was statistically justified for 18 of the 60 structures) with main effects of genotype, age, and sex, including interaction terms. For structures where there was a significant age-by-genotype interaction, genotype differences were assessed by using the best fit linear or quadratic model to interpolate the absolute structure volumes at $130 \mathrm{~d}$ of age. Multiple comparisons were controlled using a false discovery rate (FDR) threshold of 10\% (Genovese et al., 2002). To investigate the cellular mechanism underlying the brain tissue damage, two $\mathrm{dcr}$ and two littermate control mice at $\sim 150 \mathrm{~d}$ of age were studied by histopathology. The total number of cells in the granular cell layer of the hippocampus was counted in six random samples with a frame size of $100 \times 100 \mu \mathrm{m}$ on one coronal brain slice. The number of cells was analyzed using a linear mixed-effects model with group ( $d c r$, control) as a fixed effect and mouse ID as a random effect to account for variation between mice.

At 8-10 weeks of age, 9 dcr (5 males and four females) and 9 littermate control (5 males and four females) mice were trained on the Barnes maze, a test of spatial learning and memory (Barnes, 1979). The mice then repeated the training 1 month later. These time points were chosen to capture the onset of symptoms and before degeneration and overt abnormal behavior (i.e., lethargy). Primary latency (Harrison et al., 2006), the time to the first encounter with the escape hole and path length were recorded during each training trial. To assess any differences in behavioral outcomes between groups, for each training session we perform an ANOVA on a linear mixed-effects model with trial and genotype as fixed effects and allowing for interaction between the two. Mouse ID was treated as a random factor to account for variation between mice. If there was a significant trial-by-genotype interaction, we performed post hoc $t$ tests to investigate the performance of each group for each session. We also tested to see whether there were any differences in behavior between sessions for each group, using an ANOVA on a 
linear mixed-effects model with trial and session as the fixed effects and mouse ID as the random variable. For the probe trial, the same models were used to test for differences in time spent in each quadrant (normalized to yield percentage time) except quadrant was substituted for trial. A value of $p<0.05$ was taken to be significant.

To investigate the anatomy of the cerebrovasculature, $3 \mathrm{D}$ datasets were acquired for $17 \mathrm{dcr}$ mice ( 6 males and 11 females) and 13 control mice ( 5 males and 8 females) using micro-CT at $53-153 \mathrm{~d}$ of age. The data were analyzed using a two-way ANOVA to evaluate the effects of genotype and age. A value of $p<0.05$ was taken to be significant.

An ex vivo MRI study was performed to determine whether the neuroanatomical changes detected in the $d c r$ mice were recapitulated in the $\mathrm{Mrpl}^{+/-}$mice. At 12 weeks of age, $24 \mathrm{Mrpl}^{+/-}$mice (12 males and 12 females) and 19 littermate controls (10 males and 9 females) were anesthetized with ketamine/xylazine, and a transcardiac perfusion was performed as previously described (Cahill et al., 2012). To detect neuroanatomical differences between $\mathrm{Mrpl3}^{+/-}$and control mice, we used a linear model for each structure and at every voxel with the main effects of group, sex, and group-by-sex interaction $(\mathrm{FDR}=10 \%)$.

In vivo and ex vivo magnetic resonance imaging. MRI was conducted on a multichannel $7.0 \mathrm{~T}, 40$-cm-diameter bore magnet (Varian) equipped for imaging multiple mice simultaneously (Dazai et al., 2011). For in vivo imaging, mice were imaged using $0.7-1.0 \%$ and $1.0-1.2 \%$ isoflurane for the $d c r$ and control mice, respectively. The isoflurane concentration was chosen to maintain a constant breathing rate of $\sim 40-60$ breaths/minute. The mice were kept warm during the scan using warm air, with the body temperature and breathing continuously monitored throughout the imaging. The in vivo imaging protocol consisted of a T2-weighted, 3D fast spin-echo with the following parameters: $\mathrm{TR}=1800 \mathrm{~ms}$; $\mathrm{TE}_{\text {eff }}=40 \mathrm{~ms}$; echo train length $=12$; field-of-view $=3.5 \times 4.2 \times 2.1 \mathrm{~cm}$; matrix size $=$ $280 \times 336 \times 168$; isotropic image resolution $=125 \mu \mathrm{m}$. The scan time was $2 \mathrm{~h}$ and $20 \mathrm{~min}$. The ex vivo imaging protocol consisted of a T2-weighted, $3 \mathrm{D}$ fast spin-echo using a cylindrical $\mathrm{k}$-space acquisition with $\mathrm{TR}=350 \mathrm{~ms} ; \mathrm{TE}=12 \mathrm{~ms}$; echo train length $=6$; four averages; field-ofview $=2.0 \times 2.0 \times 2.5 \mathrm{~cm}$; matrix size $=504 \times 504 \times 630$; isotropic image resolution $=40 \mu \mathrm{m}$ (Spencer Noakes et al., 2017). The scan time was $14 \mathrm{~h}$.

The in vivo and ex vivo images were analyzed separately but followed similar registration procedures. Consensus average brain images were derived from the 74 individual in vivo $\mathrm{MR}$ images and the 43 ex vivo MR images using an automated image registration approach (Lerch et al., 2011a). The Jacobian determinants provided an estimate of the local volume changes at every voxel in the brain. A segmented atlas, with 60 labeled structures for the in vivo images and 182 structures for the ex vivo images, was registered to the average image to calculate the volumes of the brain structures in each image (Dorr et al., 2008). The lesion volume was determined by manual segmentation using Display (MINC tool kit, McConnell Brain Imaging Center).

Histology. Fixed brains were removed from the skull, paraffin embedded, and sectioned $(4 \mu \mathrm{m})$ on the coronal plane. Tissue sections were stained with hematoxylin and eosin, and the slides were digitized using a slide scanner at $40 \times$ resolution.

Behavioral analysis. Briefly, the Barnes maze consists of a circular table with 40 equidistant holes located at the periphery and one "escape box" (Scholz et al., 2015). The maze is surrounded by four spatial cues, and mice are tracked by Ethovision XT software (Noldus Information Technology). After a $60 \mathrm{~s}$ habituation trial, mice were trained on six trials per day for 2 consecutive days. Each trial ended when the mouse either entered the escape box or after $180 \mathrm{~s}$ had passed and they were guided to the correct hole. On day 3, a probe trial, where the escape box was replaced with a shallow well, was run for $90 \mathrm{~s}$.

Genome sequencing and confirmation of insertion. Genomic DNA was extracted from a homozygous $d c r$ mouse and sent to Nimblegen for array capture (Nimblegen). Targeted resequencing was conducted on the $d c r$ genetic interval on chromosome 9 as well as $\sim 1$ Megabase $(\mathrm{Mb})$ upstream and downstream of this region (9:103.3-106.3 Mb, MGSCv37, $\mathrm{mm} 9$ ). The genomic DNA was then fragmented, hybridized to the array, and eluted. Following paired-end sequencing on Illumina HiSeq 2000 at the Vanderbilt University Microarray Shared Resource, the sequence reads (100 bp in length) were analyzed using Galaxy. In addition, reads were aligned to a reference genome, and the alignment was visualized using Savant Genome Browser (Fiume et al., 2010). The Velvet de novo assembler (Zerbino and Birney, 2008) was used to identify long continuous sequences that did not match the reference genome and to look for structural variants. After identification of a novel insertion, primers were designed using an Ensembl sequence to bracket the site of interest (TCTCGAGAGTCAGCTCATAGAGACA and CCCCGCAGAGAC TCATTACCT), tested in two $d c r$ and two control mice, and then assembled de novo.

To establish causality, a quantitative complementation test (Hawley and Gilliland, 2006) was performed by breeding heterozygous male $d c r$ mice with heterozygous female $\mathrm{Mrpl} 3$ mice. Four breeding pairs were used, and "failure to complement" was defined as the absence of compound heterozygotes after genotyping at least 50 pups.

Mitochondrial enzyme activity. To investigate respiratory chain defects, mouse brains were frozen in liquid nitrogen and stored at $-80^{\circ} \mathrm{C}$ until use. Samples were collected at 52, 120, and $143 \mathrm{~d}$ for $d c r$ and control mice $(n=5-6 /$ group/age). Tissue was homogenized in $10 \times \mathrm{w} / \mathrm{v}$ buffer $(10 \mathrm{mg} / \mathrm{ml}$ fatty acid free bovine serum albumin, $0.17 \mathrm{mg} / \mathrm{ml}$ dithiothreitol, $1 \mathrm{mg} / \mathrm{ml} \mathrm{NAD}, 11 \mathrm{~mm}$ potassium phosphate, $3 \mathrm{~mm} \mathrm{MgCl}_{2}$, and $1 \mathrm{~mm}$ EDTA, pH 7.4) for 30 strokes using a 7 $\mathrm{ml}$ Wheaton glass homogenizer. Homogenates were then centrifuged at $3000 \times g$ for $10 \mathrm{~min}$ at $4^{\circ} \mathrm{C}$. Supernatants were frozen in liquid nitrogen, and enzyme activities assayed the same day. Respiratory chain enzymes were measured as described for Complex I+III, Complex II+III, Citrate synthase (Moreadith et al., 1984), and Complex IV (Glerum et al., 1988).

Phenotyping data collection. The $\mathrm{Mrpl}^{+/-}$mice (5-10 males and 59 females) underwent high-throughput adult phenotyping based on the TCP pipeline described on the International Mouse Phenotyping Resource of Standardised Screens (IMPReSS) database (https://www. mousephenotype.org/impress). The phenotyping tests include body weight, combined SHIRPA and dysmorphology, body composition, clinical chemistry, tests of anxiety and exploratory behavior (open field), neuromuscular function (grip strength), startle reflex (acoustic startle and prepulse inhibition), learning and memory (fear conditioning), energy metabolism, and heart function. Information about the experimental design, equipment, age of the mice, significant metadata parameters, and data quality control are available on the IMPReSS website. The control data were available from C57BL/6NCrl mice (5-10 males and 59 females) tested during the same time period.

Micro-CT imaging and vascular segmentation. Detailed methods for preparing the cerebrovasculature for micro-CT imaging have been previously described (Chugh et al., 2009). Briefly, mice were anesthetized with an intraperitoneal injection of ketamine/xylazine and $200 \mathrm{U}$ of heparin. The inferior vena cava and descending aorta were ligated, and a needle was inserted into the left ventricle. Warm heparinized PBS was perfused at $50 \mathrm{mmHg}$, followed by Microfil (Flow Tech) at $150 \mathrm{mmHg}$. The Microfil was allowed to polymerize at $30 \mathrm{mmHg}$, approximating normal capillary pressure. The dissected skulls were fixed for $24 \mathrm{~h}$ at $4^{\circ} \mathrm{C}$ in $10 \%$ buffered formalin phosphate, decalcified in $8 \%$ formic acid at room temperature for $48 \mathrm{~h}$ and mounted in $1 \%$ agar. Using a GE eXplore Locus SP Specimen micro-CT scanner, the x-ray source was set at $80 \mathrm{kV}$ and $80 \mu \mathrm{A}$, and the specimen was rotated $360^{\circ}$, generating 720 views in $2 \mathrm{~h}$, which were reconstructed into data blocks with a $20 \mu \mathrm{m}$ voxel size.

All of the micro-CT images were registered to a common space using a 3D MRI anatomic brain atlas (Dorr et al., 2008) and vascular landmarks as previously described (Chugh et al., 2009). Each image was manually segmented to exclude extracerebral vessels using Display. The cerebrovasculature was automatically segmented, identifying vessel structures and their connectivity using an algorithm previously described in detail (Ghanavati et al., 2014). Measurements of total vessel segment numbers, total length of vessel segments, and diameter distributions were extracted from the tubular models. Detection of vessels with a diameter $<60 \mu \mathrm{m}$ was unreliable, and therefore the terminal segments of the vascular tree were pruned to $60 \mu \mathrm{m}$ to improve data consistency. 


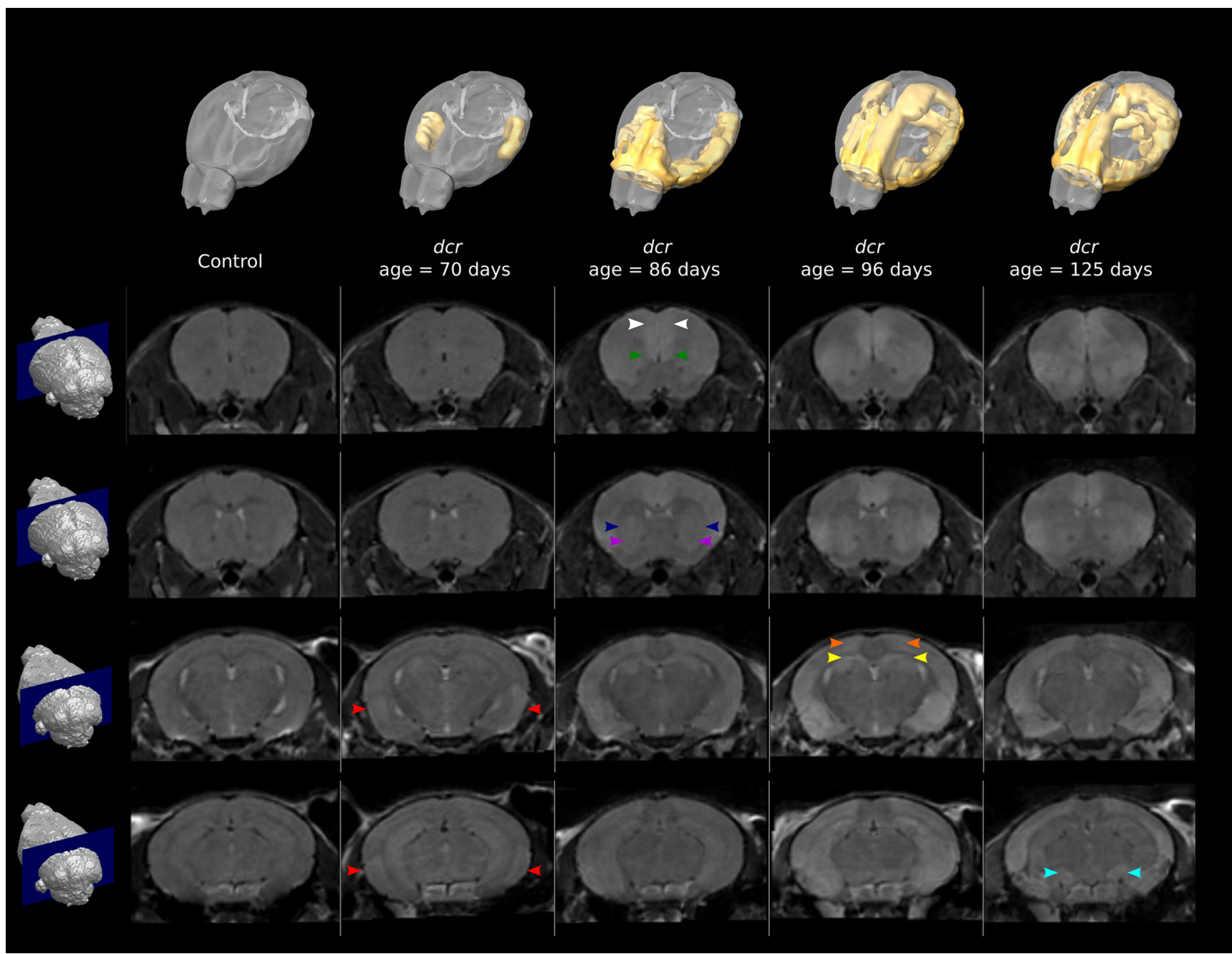

Figure 1. A time course examining the progression of neurodegeneration in the der mouse. Top row, Manual segmentation of the hyperintensities demonstrate that the lesion is bilaterally symmetric and progressive. Bottom rows, Representative coronal sections of in vivo T2-weighted MR images. The lesion starts in the piriform and entorhinal cortices (red arrowheads at $70 \mathrm{~d}$ ) and progresses to the limbic area (green arrowheads at $86 \mathrm{~d}$ ), cingulate cortex (white arrowheads at $86 \mathrm{~d}$ ), striatum (dark blue arrowheads at $86 \mathrm{~d}$ ), nucleus accumbens (purple arrowheads at $86 \mathrm{~d}$ ), motor cortex (orange arrowheads at $96 \mathrm{~d}$ ), hippocampus (yellow arrowheads at $96 \mathrm{~d}$ ), and substantia nigra (light blue arrowheads at $125 \mathrm{~d}$ ).

\section{Results}

\section{MRI-detectable pattern of neurodegeneration}

Using in vivo T2-weighted MRI, the time course of the decrepit phenotype was investigated (Fig. 1). Bilateral and symmetrical hyperintensities were observed starting in the piriform and entorhinal cortices at $70 \mathrm{~d}$ of age; spreading to the limbic area, cingulate cortex, striatum, and nucleus accumbens at $86 \mathrm{~d}$; to the motor cortex and hippocampus at $96 \mathrm{~d}$; and finally to the substantia nigra at $125 \mathrm{~d}$. In the MR images at later ages, the lesion takes on a patchy appearance with abnormal elongated hypointensities. The areas of lesion are consistent with regions of multifocal necrosis identified using histologic techniques (Fig. 2a,b), confirming that the MRI hyperintensities correspond to areas of cell death (see below). The progressive degeneration is reproducible and consistent with the results from histopathology detailed in the study by Sproule et al. (2010); however, 3D MRI detected abnormal tissue intensity at earlier time points and in regions that were not previously identified by histology such as the striatum.

\section{Neuroanatomical volume changes}

A significant age-by-genotype interaction demonstrated that many of the brain structures had an altered trajectory of growth from 50 to $150 \mathrm{~d}$ of age in the $d c r$ compared with control mice $(F$-statistic $=2.89,10 \%$ FDR; Fig. 3). When we included sex as a covariate, there was no effect on the statistical significance. The differences were found throughout the brain including gray matter structures (amygdala, bed nucleus of stria terminalis, entorhinal cortex, frontal lobe, occipital lobe, parieto-temporal lobe, dentate gyrus, hippocampus, hypothalamus, lateral septum, mammillary bodies, medial septum, pre-post-parasubiculum, and thalamus), white matter structures (anterior commissure, corpus callosum, fimbria, habenular commissure, mammilothalamic tract, optic tract, and stria medullaris), the subependymale zone/rhinocele of the olfactory region, and in the brainstem (cuneate nucleus and superior olivary complex). In the control mice, the volume of most of the brain structures remained constant from 50 to $150 \mathrm{~d}$ of age while the volume in the $\mathrm{dcr}$ mice decreased. The frontal lobe, occipital lobe, parieto-temporal lobe, cuneate nucleus, medial septum, and superior olivary complex increased in the $\mathrm{dcr}$ mice. The volume of the anterior commissure, fimbria, hypothalamus, lateral septum, subependymale zone/rhinocele, and thalamus increased with age in the controls and either remained constant (anterior commissure, corpus callosum, fimbria, hypothalamus) or decreased (lateral septum, subependymale zone/rhinocele, thalamus) in the $d c r$ mice. 

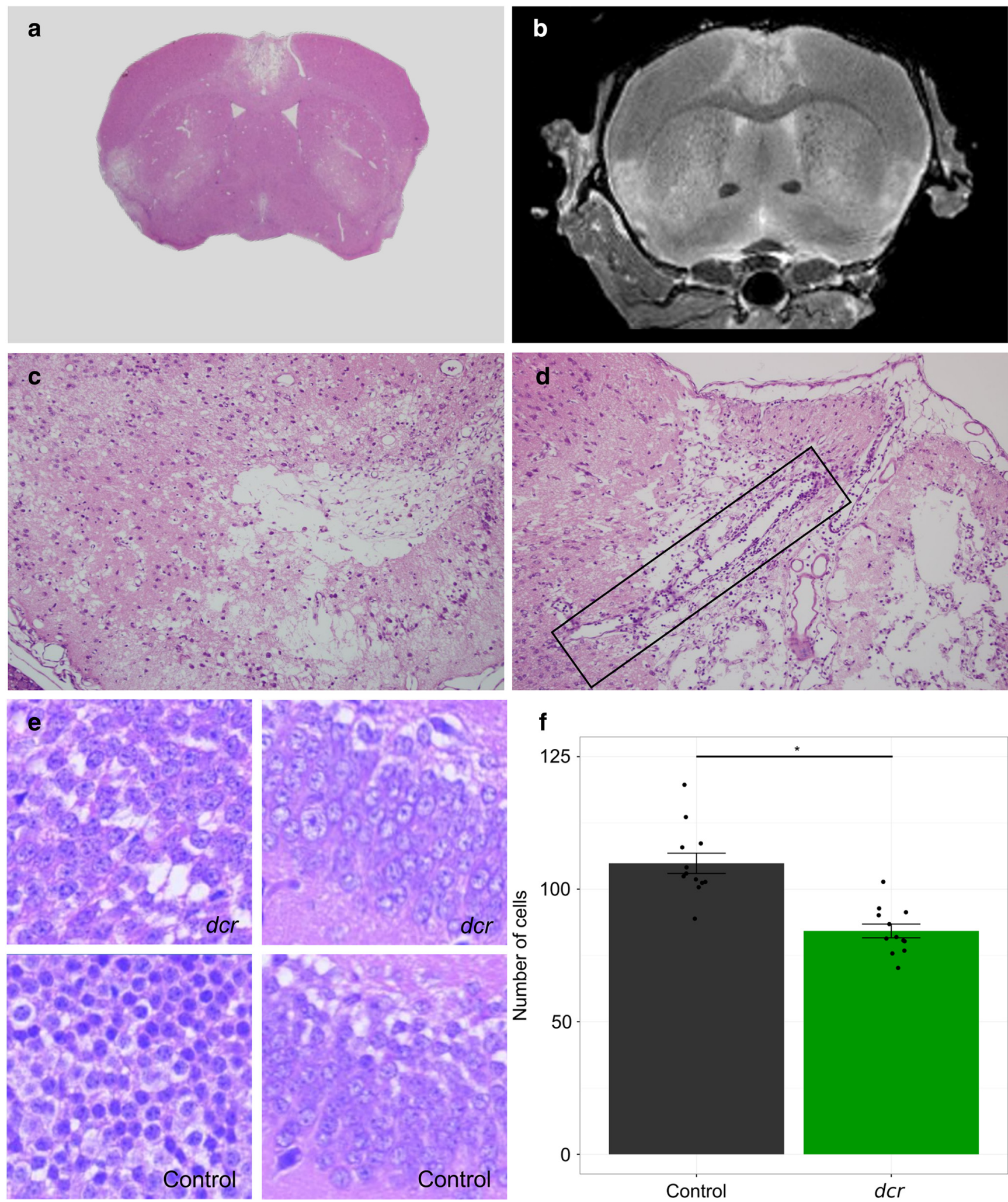

Figure 2. Pathology in the brain of $d c r$ mice at late stages of the disease. $\boldsymbol{a}, \boldsymbol{b}$, The tissue loss in a hematoxylin and eosin-stained coronal section (a) corresponds to the hyperintensities in an ex vivo T2-weighted MR image (b). $\boldsymbol{c}, \boldsymbol{d}$, There was complete vacuolization of areas of the tissue in the entorhinal cortex (c) and cingulate cortex (d; $20 \times$ resolution). Dilated blood vessels (within the rectangle in $\boldsymbol{d}$ ) were also present. $\boldsymbol{e}$, Representative $100 \times 100 \mu \mathrm{m}$ frames from the granular cell layer of the hippocampus in the $d c r$ mice (top) and control mice (bottom). $f$, The number of cells in the granular cell layer were significantly decreased in the $d c r$ mice (green bar) compared with controls (black bar). The error bars represent the SEM. $n=2 /$ group. ${ }^{*} p=0.03$. 

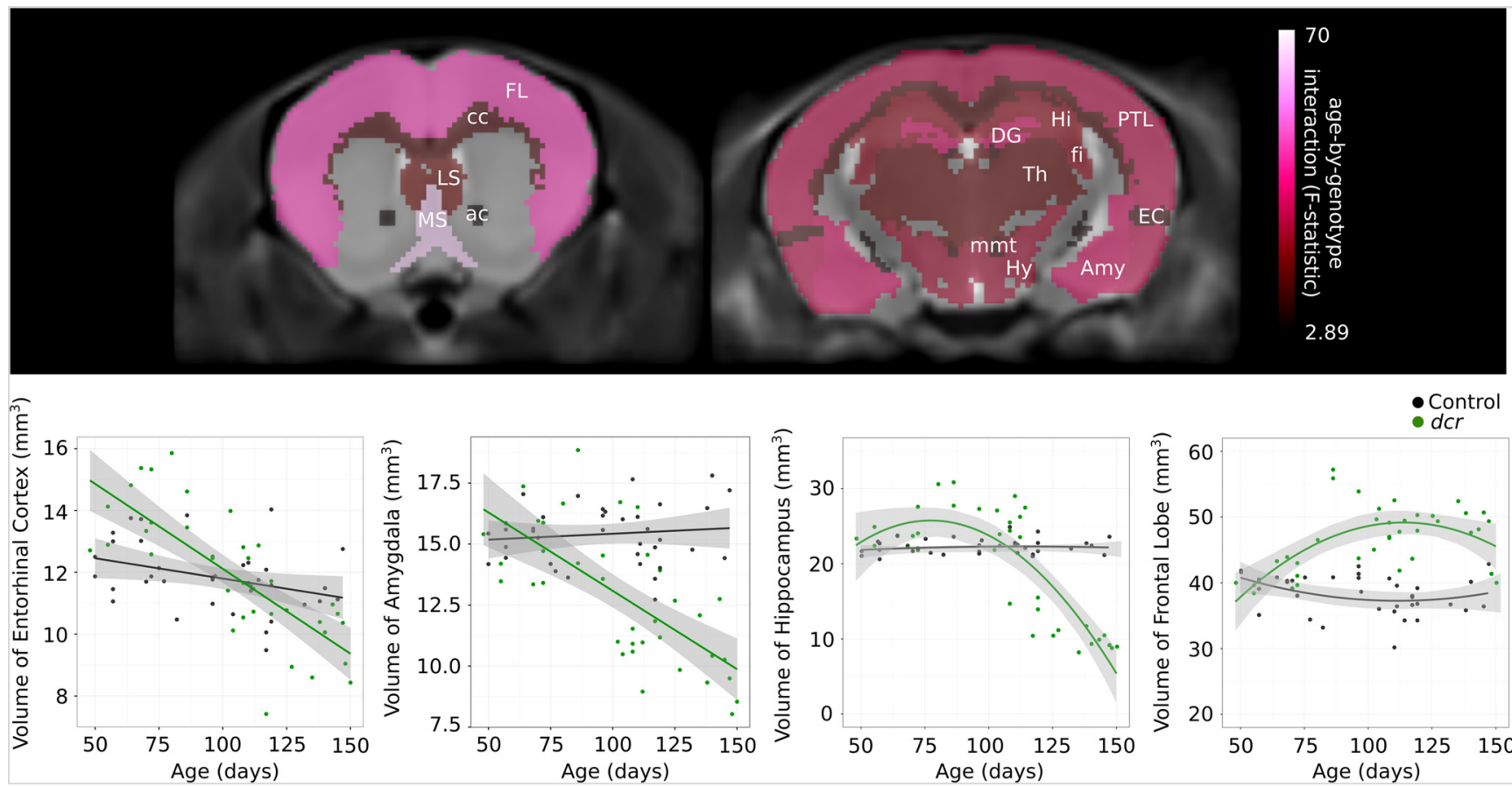

Figure 3. Differences in growth trajectory by brain structure. Top row, Coronal MRI slices showing the structures where there was a significant age-by-genotype interaction ( $F$ statistic $=2.89,10 \%$ FDR). Bottom row, The structure volume change with age is shown for selected structures for $d c r$ (green) and control (black) mice ( $n=37 /$ group). The entorhinal cortex and amygdala were fit using a linear model, and the hippocampus and frontal lobe were fit using a quadratic model. The shaded gray area represents the $95 \%$ confidence interval. ac, Anterior commissure: pars anterior; Amy, amygdala; cc, corpus callosum; DG, dentate gyrus of hippocampus; EC, entorhinal cortex; fi, fimbria; FL, frontal lobe; Hi, hippocampus; Hy, hypothalamus; PTL, parieto-temporal lobe; LS, lateral septum; mmt, mammilothalamic tract; MS, medial septum; Th, thalamus.

Applying the best fit linear or quadratic model used to fit the regional growth over time to the structures that showed significant alterations, the volume differences between $d c r$ and control mice at $130 \mathrm{~d}$ were interpolated, and the results are summarized in Table 2. The magnitude of the volume changes was dramatic, with the hippocampus and dentate gyrus showing a volume decrease of $33 \%$ and $43 \%$, respectively, in the $d c$ mice compared with controls. Interestingly, areas of the cerebral cortex were 20$33 \%$ larger in the $d c r$ mice.

\section{Cell death is the central mechanism of the observed neurodegeneration}

A pathology analysis of two $d c r$ and two control brains was performed by a veterinary pathologist at The Center for Modeling Human Disease who was blinded to the genotype of the animals. In the $d c r$ mice, multiple focally extensive and well demarcated areas of necrosis were identified in the cingulate, piriform and entorhinal cortices; the amygdala; and the hippocampus. Prominent vacuolation of the neuropil was noted (Fig. $2 c, d$ ), with replacement by necrotic cells with karyorrhectic debris, reactive astrocytes, macrophages, and lymphocytes. The blood vessels were found to be dilated and to have hypertrophic endothelial cells (Fig. 2d). To understand the cellular bases of the neuroanatomical volume changes, we examined the number of cells in the granular cell layer of the hippocampus (Fig. 2e). The $d c r$ mice had significantly fewer cells than the controls $(p=0.03)$, consistent with cell death (Fig. 2f).

\section{Impaired learning and a progressive loss of memory}

Mice were trained and tested on the Barnes maze during adulthood at two time points, separated by 1 month. For both training sessions, the performance differed between the $d c r$ and control
Table 2. The differences in absolute volume (mean \pm confidence interval) of the $d c r$ compared with control mice at $130 \mathrm{~d}$ of age ( $n=37 /$ group)

\begin{tabular}{lc}
\hline Structure & Difference in absolute volume at 130 d of age (\%) \\
\hline Amygdala & $-28 \pm 2$ \\
Anterior commissure: pars anterior & $-8 \pm 1$ \\
Anterior commissure: pars posterior & $26 \pm 3$ \\
Bed nucleus stria terminalis & $-11 \pm 1$ \\
Cerebral cortex: entorhinal cortex & $-8 \pm 1$ \\
Cerebral cortex: frontal lobe & $29 \pm 2$ \\
Cerebral cortex: occipital lobe & $33 \pm 3$ \\
Cerebral cortex: parieto-temporal lobe & $20 \pm 1$ \\
Corpus callosum & $4 \pm 1$ \\
Cuneate nucleus & $58 \pm 15$ \\
Dentate gyrus of hippocampus & $-43 \pm 6$ \\
Fimbria & $-22 \pm 1$ \\
Habenular commissure & $-35 \pm 8$ \\
Hippocampus & $-33 \pm 4$ \\
Hypothalamus & $-14 \pm 1$ \\
Lateral septum & $-23 \pm 2$ \\
Mammillary bodies & $-14 \pm 2$ \\
Mammilothalamic tract & $-18 \pm 2$ \\
Medial septum & $70 \pm 7$ \\
Optic tract & $-4 \pm 1$ \\
Pre-post-parasubiculum & $-18 \pm 2$ \\
Stria medullaris & $-15 \pm 1$ \\
Subependymale zone/rhinocele & $-32 \pm 4$ \\
Superior olivary complex & $19 \pm 2$ \\
Thalamus & $-13 \pm 1$ \\
\hline &
\end{tabular}

The volumes were interpolated using the best fit linear or quadratic models fit to the growth trajectories for the structures that showed significant differences between groups.

mice, with the control mice approaching the target box quicker $(p=0.0007$ and $p<0.0001$ for the initial training session and the follow-up training session, respectively; Fig. $4 a)$. The control mice performed differently between the two sessions $(p<0.0001)$, with 
the difference in primary latency with trial observed in the first session $(p<0.0001)$ absent in the second session $(p=0.2)$. In contrast, the $d c r$ mice showed improved performance with trials at both training sessions $(p<0.0001$ and $p<0.0001$ for the initial training session and the follow-up training session, respectively); however, there was no difference in the performance between sessions $(p=0.2)$. Including sex as a covariate did not alter the statistical significance.

The $d c r$ and control mice performed differently on the probe trial at both training sessions $(p=0.03$ and $p=0.02$ for the initial training session and the follow-up training session, respectively; Fig. 4b). The control mice showed a trend toward a preference for the target at the initial training session $(p=0.1)$ and a strong preference for the target during the follow-up training session $(p<0.0001)$. The $d c r$ mice showed a preference for the target at the initial training session $(p=0.002)$ and did not show any preference at the follow-up training session $(p=0.9)$. During both training sessions, the $d c r$ mice visited fewer holes during the probe session compared with controls $(p=0.01$ and $p=0.002$ for the initial training session and the follow-up training session, respectively).

\section{Novel insertion identified in an intron of the Mrpl3 gene}

Next-generation sequencing was performed on genomic DNA isolated from the dcr genetic interval on chromosome 9 as well as $1 \mathrm{Mb}$ flanking regions. Seven sequence variations (i.e., single nucleotide polymorphisms) were detected; however, they were shown to be differences from the reference genome when PCR amplified and resequenced using Sanger methods, even from wild-type mice in the $d c r$ colony. To look for structural variants (Baker, 2012), all of the reads that did not map well to the reference genome were assembled using the Velvet de novo assembler. A continuous sequence matched over most of the region of chromosome 9, but there were no pairs that spanned the region. The half-mapped pairs from each side were then assembled. Using the primers designed to bracket this region (GRCm38 chr9:105,064,197-105,064,647), we confirmed that the $d c r$ mouse contains the following insertion within introns 6-7 of the Mrpl3 gene at 105,064,438-9 (bracketing native sequence included for reference, insertion underlined), as follows:

GGTGGGCGACCCCAGTGACTACTGCAGTTTGACTC CAGGTTCTTTTTTTTTTTTTTTTTTTTTTTTTTTTTTTT TTTTTTTTTTTTTTTTTTTTTTTTTTTTTTTTTTTTTTT TTTTTTTTTTTGATTCTTTATTTATTTATTTCAGGAA CACCTCGATGCTCGACTCCAGGTTCTTTCTAGTCAC CGGGAGT

The minimum length of the insertion is $133 \mathrm{bp}$, but the exact length could not be determined because both the forward and reverse priming failed with the $\mathrm{T}$ repeat region. Based on the band size estimated by gel electrophoresis, we anticipate that the insertion is not much larger because it increases an expected $451 \mathrm{bp}$ product to $\sim 584 \mathrm{bp}$.

\section{Mrpl3 knock-out mice are embryonic lethal}

After identification of the novel insertion in the Mrpl3 gene, we generated Mrpl3 knock-out mice to ascribe causality of the decrepit phenotype to the Mrpl3 insertion using a quantitative complementation test and to investigate the phenotype of the $\mathrm{Mrpl3}^{-1-}$ mice. Complete knockout of the Mrpl3 gene resulted in early embryonic lethality with no homozygotes at embryonic day 12.5 (E12.5; $n=35,7$ wild-type, 28 heterozygotes, 0 homozygotes) or E9.5 ( $n=28,8$ wild-type, 20 heterozygotes, 0 homozygotes). In addition, there was no evidence of any homozygotes postimplantation (no homozygous resorptions at E9.5). From the breeding pairs of heterozygous $\mathrm{Mrpl3}$ and mice heterozygous for the $d c r$ mutation, we genotyped 66 pups (from 10 litters) and found that the homozygous mice were lethal before P14, indicating a failure to complement.

The heterozygous Mrpl3 mice underwent a battery of phenotyping tests (Fig. 5). Mrpl3 $3^{+/-}$mice have a normal life span and show no overt phenotype. The only sex difference was found in the following body weight data: the female $\mathrm{Mrpl}^{+/-}$mice had a significantly slower growth rate than the controls $(p=0.0003)$. In the open field test, the $\mathrm{Mrpl}^{+/-}$mice spent significantly less time in the center than the controls $(p=0.005)$, although the distance they traveled was similar, suggesting an increase in overall anxiety levels. This difference was maintained throughout the $20 \mathrm{~min}$ of testing, indicating that the anxiety levels were elevated in both the exploratory and habituation phases of the test. The heart rate was significantly elevated in the $\mathrm{Mrpl}^{+/-}$mice compared with controls $(p=0.03)$, while none of the other measurements indicated abnormal cardiovascular physiology. The levels of several chemicals in the blood were abnormal, including a decrease in creatinine $(p=0.0009)$, an increase in calcium $(p=$ 0.0001 ), and a trend toward increases in the liver enzymes 

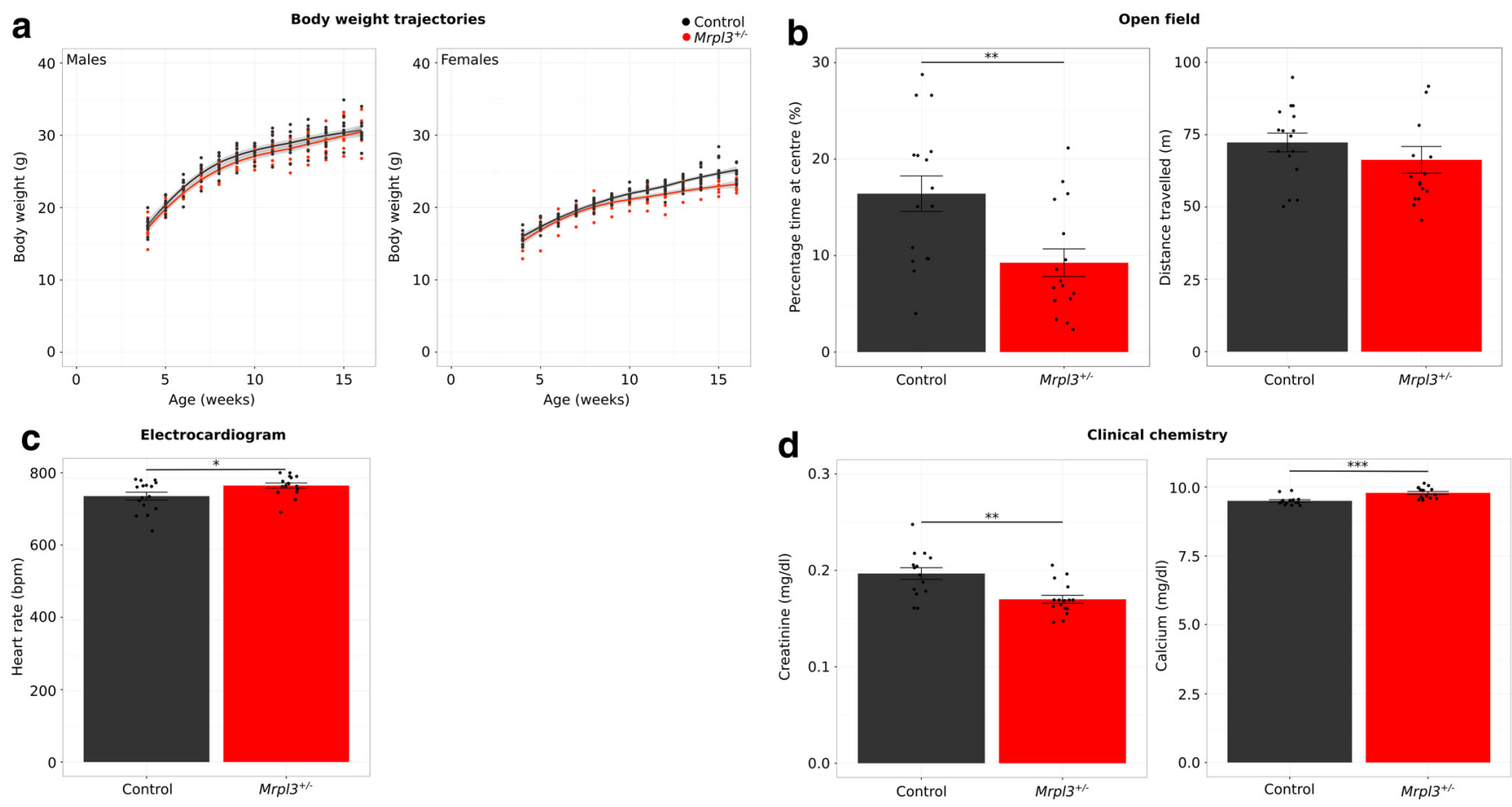

Figure 5. Adult phenotyping for the $M r p / 3^{+/-}$mice (red) and control mice (black). $\boldsymbol{a}$, The female Mrp/3 ${ }^{+/-}$mice had a slower growth trajectory than the controls. $\boldsymbol{b}$, The Mrp/3 $3^{+/-}$mice spent less time in the center of the open field test despite traveling a similar distance as the controls. $\boldsymbol{c}$, The Mrp/ $3^{+/-}$mice had an elevated heart rate compared with controls. $\boldsymbol{d}$, Creatinine was decreased and calcium was increased in the $\mathrm{Mrp} / 3^{+/-}$mice compared with controls. The error bars represent the SEM. $n=16 /$ group ( 8 males, 8 females) except for body weight trajectories where $n=19$ (10 males, 9 females). ${ }^{*} p<0.05,{ }^{* *} p<0.005,{ }^{* * *} p<0.0001$.

alkaline phosphatase $(p=0.1)$ and alanine aminotransferase $(p=0.1)$ compared with controls. Interestingly, there were abnormalities in the eye morphology of the $\mathrm{Mrpl}^{+/-}$mice with 6 of 15 showing evidence of either retinal dysplasia or severe retinitis pigmentosa. All of the control mice had normal eye morphology. Mutations in $>60$ genes are known to be associated with retinitis pigmentosa (https://ghr.nlm.nih.gov/condition/ retinitis-pigmentosa); however, $\mathrm{Mrpl3}$ has not yet been implicated. At 12 weeks of age, a high-resolution ex vivo MRI study showed that there were no significant neuroanatomical differences between the $\mathrm{Mrpl}^{+/-}$mice and littermate controls at a $10 \%$ FDR threshold. Including sex as a covariate did not alter the statistical significance.

Mrpl3 is a nuclear gene that encodes 1 of 48 proteins comprising the large subunit of the mitochondrial ribosome (OMIM\# 607118). Thus, we decided to determine whether mitochondrial function is affected at three time points during the life span of the $d c r$ homozygous mice (before onset of symptoms, after significant neurodegeneration, and shortly before death). Mitochondria were prepared from brain tissue, and the activity of the mitochondrial respiratory chain complexes was measured. There was no effect of age when it was included as a covariate. Figure 6 shows that there were no differences in the activities of NADH:cytochrome $c$ reductase [complex I+III $(\mathrm{CI}+\mathrm{CIII})]$, succinate:cytochrome $c$ reductase [complex II + III $(\mathrm{CII}+\mathrm{CIII})$ ], and cytochrome oxidase [complex IV (CIV)]. There was a significant increase in the citrate synthase (CS) activity for the $\mathrm{dcr}$ mice compared with controls $(p=0.003)$. Citrate synthase is a marker for mitochondrial integrity, and an increase can indicate mitochondrial proliferation.

\section{Hypervascularization in response to neurodegeneration}

In our previous study of the $d c r$ mice, no major anatomic abnormalities of the cerebral vasculature were observed in affected

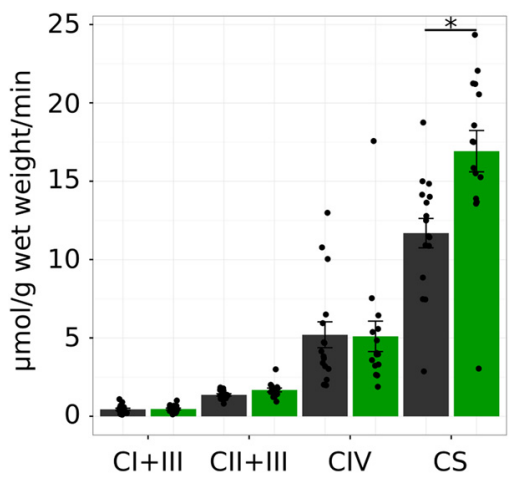

Figure 6. Activities of the mitochondrial enzymes in mouse brain tissue from $d c r$ (green bars) and control (black bars) mice. The error bars represent the SEM. $n=15-16 /$ group. ${ }^{*} p=0.003$.

mice ( $n=2$ at $100 \mathrm{~d}$ of age). Advanced 3D imaging using x-ray micro-CT (Fig. 7a,b), in combination with automated vascular segmentation, allows us to perform a detailed analysis of the cerebrovasculature over the time course of pathology. The dcr mice showed a significant difference in the total number of vessel segments with age compared with controls $(p=0.002$; Fig. $7 c)$. The increase in vessels appears to occur after $100 \mathrm{~d}$, suggesting that vascular proliferation is occurring in response to the neurodegeneration. To determine whether the increase in vasculature was associated with the injured region, the total vessel segments were divided into those found in regions prone and non-prone to lesion (based on the segmentation from a $d c r$ mouse at $125 \mathrm{~d}$ of age; Fig. 1). Hyper-vascularization in the $d c r$ mice appears to be global, increasing in both the prone and non-prone regions with age ( $p=0.01$ and $p=0.002$ respectively). When the cumulative number of vessel segments was plotted as a function of diameter for the brains $>125 \mathrm{~d}$ of age, the curve for the $d c r$ mice 

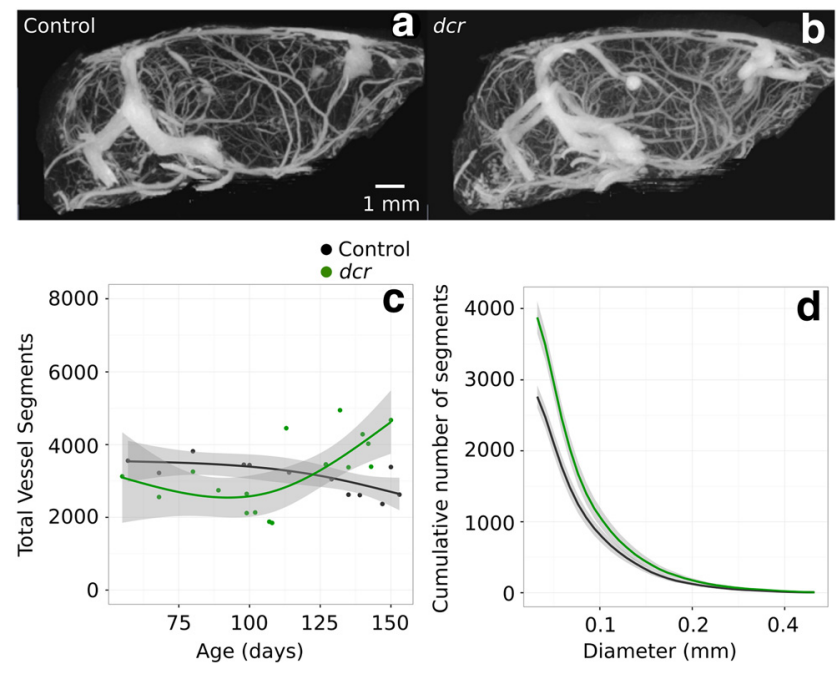

Figure 7. Number of vessel segments. $\boldsymbol{a}, \boldsymbol{b}$, Maximum intensity projection images of representative control (a) and $d c r$ cerebral vascular trees $(\boldsymbol{b})$ at $150 \mathrm{~d}$ of age. $\boldsymbol{c}$, The $d c r$ mice (green) showed an increase in the total number of vessel segments after the onset of neurodegeneration ( $n=17 \mathrm{dcr}$ mice; $n=13$ control mice). The shaded gray area represents the $95 \%$ confidence interval. $\boldsymbol{d}$, The number of vessel segments greater than a given diameter for the $d c r$ mice (green) compared with control mice (black) $>125 \mathrm{~d}$ of age shows that the increase in vessels occurs in the small-diameter vessels ( $n=7 /$ group). The data are presented as the mean \pm SEM.

rises more steeply toward the small diameter range compared with controls (Fig. $7 d$ ). This indicates that the increase in the number of cerebral vessels is occurring primarily in the small vessels.

\section{Discussion}

Using structural variant analysis, we identified a novel insertion within an intron of $\mathrm{Mrpl3}$ that results in reproducible adult-onset neurodegeneration in mice. The discovery of this nuclear genetic mutation was made possible by the use of next-generation sequencing technologies and de novo sequence assembly. Creation of a $\mathrm{Mrpl3}$ knockout confirmed by complementation testing that the $d c r$ allele of $\mathrm{Mrpl} 3$ results in a loss of $\mathrm{Mrpl} 3$ function. In our previous attempt to identify the causative mutation (Sproule et al., 2010), the insertion was most likely missed because it would have been categorized as failing to map to the interval. To date, the function of the Mrpl3 gene is unknown; however, early embryonic lethality in the Mrpl3 knock-out mice emphasizes its critical role in development. For the mice investigated in this study, the difference in the severity of the phenotype (embryonic lethality in the complete knockout, premature death in the $d c r$ mice, and normal life span in $\mathrm{Mrpl3}^{+/-}$mice) is likely due to differences in the expression levels, with the intronic insertion in the $d c r$ allele causing only a partial loss of function. Mutation of the Mrpl3 gene has been reported in two human case studies (Galmiche et al., 2011; Bursle et al., 2017). The pathology of these cases was heterogeneous, including structural brain abnormalities, hypertrophic cardiomyopathy, psychomotor retardation, liver dysfunction, developmental delay, and an assembly problem with the respiratory chain complexes. While these phenotypes are partially recapitulated in the $\mathrm{dcr}$ and $\mathrm{Mrpl}^{+/-}$mice (i.e., lethargy in the $\mathrm{dcr}$ mice and elevated heart rate and abnormal liver enzymes in the $\mathrm{Mrpl}^{+/-}$mice), the absence of reported neurodegeneration in the human cases means the $d c r$ mouse model may represent a heretofore undiagnosed human disease and supports evaluating non-protein-coding segments of genomes during diagnostic efforts.

$3 \mathrm{D}$ in vivo $\mathrm{T} 2$-weighted imaging revealed abnormal brain tissue, consistent with a progressive loss of neurons, and significant neuroanatomical structure volume differences in $\mathrm{dcr}$ mice compared with controls. Immunohistochemistry revealed significant cell death in the affected brain regions in the $d c r$ mice. A striking finding of this study is the large decrease in hippocampal volume over the life span of the $d c r$ mice. Our observations of impaired performance in the Barnes maze are supported by the well established involvement of the hippocampus in learning and spatial memory (McDonald and White, 1994; Lerch et al., 2011b). While the $d c r$ mice showed a deficit in learning even at the onset of symptoms, they preserved the ability to learn. Similar to neurodegenerative diseases, the $d c r$ mice showed a progressive loss of memory. All of the affected brain regions are part of a collection of structures termed the limbic system (Rajmohan and Mohandas, 2007), and the lesion follows the neuronal connectivity of the regions, specifically along the Papez circuit (Papez, 1937; Shah et al., 2012). The lesion appears to progress primarily along the perforant pathway from the entorhinal cortex to the hippocampal formation (van Groen et al., 2003). The limbic system supports a variety of brain functions including memory formation and emotion, and many of the brain structures of the limbic system have been implicated in neurodegenerative diseases. For example, cortical and hippocampal atrophy are one of the hallmarks of Alzheimer's disease (Apostolova et al., 2007; Halliday, 2017); degeneration in Parkinson's disease typically occurs in the substantia nigra (Rinne et al., 1989), basal ganglia, amygdala, and thalamus (de la Monte et al., 1989); and neuropathological changes in Huntington's disease are found in the striatum (Rosas et al., 2001), cortex (Rosas et al., 2002), amygdala, nucleus accumbens, brainstem, and hippocampus (Rosas et al., 2003). While we did not find evidence supporting a deficit in mitochondrial function in the whole brain of $\mathrm{dcr}$ mice, it may be that the structures that comprise the limbic system have higher energy requirements and are more sensitive to metabolic perturbations, contributing to subsequent neurodegeneration.

In summary, we present a spontaneous mutation in a mitochondrial-associated gene that results in progressive neurodegeneration and premature death. The $d c r$ mouse model recapitulates clinically relevant phenotypes including memory impairment and an adult-onset, stereotypical pattern of tissue degeneration that could serve as a new mouse model of neurodegenerative disease. This mouse model could aid in the development of new diagnostic procedures and could have implications for new therapies.

\section{References}

Apostolova LG, Steiner CA, Akopyan GG, Dutton RA, Hayashi KM, Toga AW, Cummings JL, Thompson PM (2007) Three-dimensional gray matter atrophy mapping in mild cognitive impairment and mild Alzheimer disease. Arch Neurol 64:1489-1495.

Baker M (2012) Structural variation: the genome's hidden architecture. Nat Methods 9:133-137

Barnes CA (1979) Memory deficits associated with senescence: a neurophysiological and behavioral study in the rat. J Comp Physiol Psychol 93:74104.

Behringer RR, Gersenstein M, Nagy K, Nagy A (2014) Manipulating the mouse embryo: a laboratory manual. Cold Spring Harbor, NY: Cold Spring Harbor Laboratory.

Bursle C, Narendra A, Chuk R, Cardinal J, Justo R, Lewis B, Coman D (2017) COXPD9 an evolving multisystem disease; congenital lactic acidosis, 
sensorineural hearing loss, hypertrophic cardiomyopathy, cirrhosis and interstitial nephritis. JIMD Rep 34:105-109.

Cahill LS, Laliberté CL, Ellegood J, Spring S, Gleave JA, van Eede MC, Lerch JP, Henkelman RM (2012) Preparation of fixed mouse brains for MRI. Neuroimage 60:933-939.

Chugh BP, Lerch JP, Yu LX, Pienkowski M, Harrison RV, Henkelman RM, Sled JG (2009) Measurement of cerebral blood volume in mouse brain regions using micro-computed tomography. Neuroimage 47:1312-1318.

Dazai J, Spring S, Cahill LS, Henkelman RM (2011) Multiple-mouse neuroanatomical magnetic resonance imaging. J Vis Exp 48:e2497.

de la Monte SM, Wells SE, Hedley-Whyte T, Growdon JH (1989) Neuropathological distinction between Parkinson's dementia and Parkinson's plus Alzheimer's disease. Ann Neurol 26:309-320.

Dickinson ME, Flenniken AM, Ji X, Teboul L, Wong MD, White JK, Meehan TF, Weninger WJ, Westerberg H, Adissu H, Baker CN, Bower L, Brown JM, Caddle LB, Chiani F, Clary D, Cleak J, Daly MJ, Denegre JM, Doe B, et al. (2016) High-throughput discovery of novel developmental phenotypes. Nature 537:508-514.

Dorr AE, Lerch JP, Spring S, Kabani N, Henkelman RM (2008) High resolution three-dimensional brain atlas using an average magnetic resonance image of 40 adult C57Bl/6J mice. Neuroimage 42:60-69.

Dutta S, Sengupta P (2016) Men and mice: relating their ages. Life Sci 152:244-248.

Fiume M, Williams V, Brook A, Brudno M (2010) Savant: genome browser for high-throughput sequencing data. Bioinformatics 26:1938-1944.

Galmiche L, Serre V, Beinat M, Assouline Z, Lebre AS, Chretien D, Nietschke P, Benes V, Boddaert N, Sidi D, Brunelle F, Rio M, Munnich A, Rötig A (2011) Exome sequencing identifies MRPL3 mutation in mitochondrial cardiomyopathy. Hum Mutat 32:1225-1231.

Genovese CR, Lazar NA, Nichols T (2002) Thresholding of statistical maps in functional neuroimaging using the false discovery rate. Neuroimage 15:870-878

Gertsenstein M, Nutter LM, Reid T, Pereira M, Stanford WL, Rossant J, Nagy A (2010) Efficient generation of germ line transmitting chimeras from C57BL/6N ES cells by aggregation with outbred host embryos. PLoS One 5:e11260.

Ghanavati S, Yu LX, Lerch JP, Sled JG (2014) A perfusion procedure for imaging of the mouse cerebral vasculature by X-ray micro-CT. J Neurosci Methods 221:70-77.

Ghasemi M, Brown RH (2018) Genetics of amyotrophic lateral sclerosis. Cold Spring Harb Perspect Med 8:a024125.

Glerum DM, Yanamura W, Capaldi RA, Robinson BH (1988) Characterization of cytochrome-c oxidase mutants in human fibroblasts. FEBS Lett 236: $100-104$.

Halliday G (2017) Pathology and hippocampal atrophy in Alzheimer's disease. Lancet Neurol 16:862-864.

Harrison FE, Reiserer RS, Tomarken AJ, McDonald MP (2006) Spatial and nonspatial escape strategies in the Barnes maze. Learn Mem 13:809-819.

Hawley RS, Gilliland WD (2006) Sometimes the result is not the answer: the truths and the lies that come from using the complementation test. Genetics 174:5-15.

Klein C, Westenberger A (2012) Genetics of Parkinson's disease. Cold Spring Harb Perspect Med 2:a008888.
Lerch JP, Sled JG, Henkelman RM (2011a) MRI phenotyping of genetically altered mice. Methods Mol Biol 711:349-361.

Lerch JP, Yiu AP, Martinez-Canabal A, Pekar T, Bohbot VD, Frankland PW, Henkelman RM, Josselyn SA, Sled JG (2011b) Maze training in mice induces MRI-detectable brain shape changes specific to the type of learning. Neuroimage 54:2086-2095.

McDonald RJ, White NM (1994) Parallel information processing in the water maze: evidence for independent memory systems involving dorsal striatum and hippocampus. Behav Neural Biol 61:260-270.

Moreadith RW, Batshaw ML, Ohnishi T, Kerr D, Knox B, Jackson D, Hruban R, Olson J, Reynafarje B, Lehninger AL (1984) Deficiency of the iron-sulfur clusters of mitochondrial reduced nicotinamide-adenine dinucleotide-ubiquinone oxidoreductase (complex I) in an infant with congenital lactic acidosis. J Clin Invest 74:685-697.

Papez JW (1937) A proposed mechanism of emotion. Arch Neurol Psychiatry 38:211-214.

Rajmohan V, Mohandas E (2007) The limbic system. Indian J Psychiatry 49:132-139.

Rinne JO, Rummukainen J, Paljärvi L, Rinne UK (1989) Dementia in Parkinson's disease is related to neuronal loss in the medial substantia nigra. Ann Neurol 26:47-50.

Rosas HD, Goodman J, Chen YI, Jenkins BG, Kennedy DN, Makris N, Patti M, Seidman LJ, Beal MF, Koroshetz WJ (2001) Striatal volume loss in HD as measured by MRI and the influence of CAG repeat. Neurology 57:1025-1028.

Rosas HD, Liu AK, Hersch S, Glessner M, Ferrante RJ, Salat DH, van der Kouwe A, Jenkins BG, Dale AM, Fischl B (2002) Regional and progressive thinning of the cortical ribbon in Huntington's disease. Neurology 58:695-701.

Rosas HD, Koroshetz WJ, Chen YI, Skeuse C, Vangel M, Cudkowicz ME, Caplan K, Marek K, Seidman LJ, Makris N, Jenkins BG, Goldstein JM (2003) Evidence for more widespread cerebral pathology in early HD: an MRI-based morphometric analysis. Neurology 60:1615-1620.

Scholz J, Allemang-Grand R, Dazai J, Lerch JP (2015) Environmental enrichment is associated with rapid volumetric brain changes in adult mice. Neuroimage 109:190-198.

Shah A, Jhawar SS, Goel A (2012) Analysis of the anatomy of the Papez circuit and adjoining limbic system by fiber dissection techniques. J Clin Neurosci 19:289-298.

Spencer Noakes TL, Henkelman RM, Nieman BJ (2017) Partitioning k-space for cylindrical three-dimensional rapid acquisition with relaxation enhancement imaging in the mouse brain. NMR Biomed 30:e3802.

Sproule TJ, Sled JG, Wentzell J, Wang B, Henkelman RM, Roopenian DC, Burgess RW (2010) A mouse model of heritable cerebrovascular disease. PLoS One 5:e15327.

Tanzi RE (2012) The genetics of Alzheimer disease. Cold Spring Harb Perspect Med 2:a006296.

van Groen T, Miettinen P, Kadish I (2003) The entorhinal cortex of the mouse: organization of the projection to the hippocampal formation. Hippocampus 13:133-149.

Zerbino DR, Birney E (2008) Velvet: algorithms for de novo short read assembly using de Bruijn graphs. Genome Res 18:821-829. 